\title{
Entstehung nativer, digitaler Plattformen und ihr Einfluss auf etablierte Unternehmen
}

Unternehmen, die ihr Geschäftsmodell auf einer digitalen Plattform aufbauen, wie beispielsweise Uber oder Airbnb, gewinnen zunehmend an Bedeutung. Im Gegensatz zu etablierten Unternehmen, die Produkte und Dienstleistungen mithilfe interner Ressourcen anbieten, greifen digitale Plattformen auf ein Ökosystem von externen Anbietern zurück. Beispielsweise tritt Uber hauptsächlich als Vermittler zwischen externen Fahrern und Passagieren auf und orchestriert deren Interaktion durch eine digitale Plattform. Durch sogenannte Netzwerkeffekte führt jeder weitere Fahrer in diesem Ökosystem zu einem erhöhten Wert für die Passagiere und umgekehrt. Doch wie können etablierte Unternehmen Anschluss an die Plattformökonomie finden? Um dies zu verstehen, ist es wichtig, sich die Entstehung von Plattformunternehmen bewusst zu machen. Der vorliegende Beitrag beschreibt, wie etablierte Unternehmen von der Entwicklung von Plattformunternehmen lernen können. Ein Beispiel wäre, dass Plattformunternehmen das Nutzungspotenzial neuer Technologien deutlich stärker explorieren, um sowohl Kunden als auch Partner in den Wertschöpfungsprozess einzubeziehen. 
Digitale Plattformen sind aus der heutigen Zeit nicht mehr wegzudenken und gewinnen zunehmend an Bedeutung, um wirtschaftlich erfolgreich zu sein [1]. Exemplarisch für den Wandel steht die Mobilitätsbranche, in der etablierte Unternehmen von jungen Start-ups wie Uber oder BlaBlaCar in kürzester Zeit überholt wurden. Ohne Vorwarnung sehen sich viele der etablierten Unternehmen mit gravierenden Veränderungen wie beispielsweise dem Wechsel von Mobilität als Produkt hin zu Mobilität als Service oder der Einbeziehung von Konsumenten in einer aktiven Rolle als Dienstleister konfrontiert. Getrieben wird diese Veränderung von sogenannten Plattformunternehmen, welche ihr Wertversprechen auf einer digitalen Plattform aufbauen [2].

Digitale Plattformen fassen im Kern drei Akteure zusammen [3]. Im Kern steht hierbei der Plattformbetreiber, welcher Steuerungsmechanismen implementiert, um eine gemeinsame Wertschöpfung zwischen Konsumenten und Anbietern zu ermöglichen. So koordiniert Uber als Plattformbetreiber die Interaktion zwischen Fahrgästen und Fahrern. Die Interaktion findet hierbei digital über eine Website oder Applikation als digitale Plattform statt. Der Vorteil für Uber ist, dass die Fahrer unabhängig sind und ihre eigenen Ressourcen in Form von Autos verwenden. Da Uber lediglich die Interaktionsplattform zur Verfügung stellt, findet die Wertschöpfung außerhalb des Unternehmens statt. Hierdurch entstehen sogenannte Netzwerkeffekte [4], bei denen mit jedem weiteren Fahrer der Wert für Passagiere und umgekehrt steigt. Dieser Effekt führt dazu, dass einige wenige Plattformunternehmen in kurzer Zeit globale Dominanz erreichen.

Wie der Exkurs zeigt, haben Plattformunternehmen nicht nur etablierte Unternehmen überholt, vielmehr haben sie die Grundregeln des Wettbewerbs verändert. Doch wie konnte es so weit kommen? Und wie können etablierte Unternehmen den Anschluss an die Plattformökonomie finden? Um beide Fragen zu beantworten, ist es wichtig, die Entstehung von Plattformunternehmen zu verstehen. Durch die Untersuchung von sogenannten nativen Plattformunternehmen, also Unternehmen, welche ihr Geschäftsmodell auf einer Plattform begründeten, können Rückschlüsse über den Einsatz neuer Technologien zum Aufbau eines Ökosystems gezogen werden. Parallel dazu müssen die Entwicklung und der Einsatz neuer Technologien für etablierte Unternehmen im gleichen Zeitraum betrachtet werden. Durch den anschließenden Vergleich zwischen Plattformunternehmen und etablierten Unternehmen wird deutlich, dass beide Unternehmen die gleichen Technologien verwenden. Der Vorteil nativer Plattformen entsteht also nicht durch den bloßen Einsatz neuer Technologien, sondern durch die Art und Weise der Verwendung.

\section{Entstehung digitaler Plattformen}

\section{Phase 1: Web 2.0}

Die Entwicklung des Internets legte den Grundstein für die globale Vernetzung von Individuen, Unternehmen und Organisationen. Knapp 15 Jahre

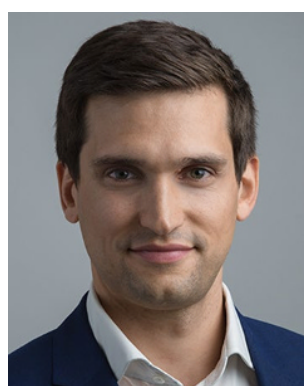

Dr. Andreas Hein ${ }^{1}(\square)$

ist Postdoktorand in der Forschungsgruppe Krcmar an der Technischen Universität München. Zusammen mit seinem Team untersucht er unter anderem Wertschöpfungsmechanismen in digitalen Plattformökosystemen.

andreas.hein@tum.de

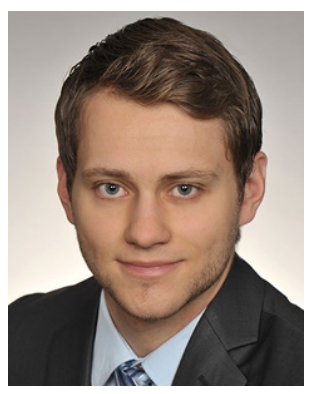

Dr. Maximilian Schreieck

ist Postdoktorand in der Forschungsgruppe Krcmar an der Technischen Universität München. Seine Themenschwerpunkte sind die Plattformökonomie, Plattformgovernance sowie die Transformation traditioneller Industrien durch digitale Plattformen. 


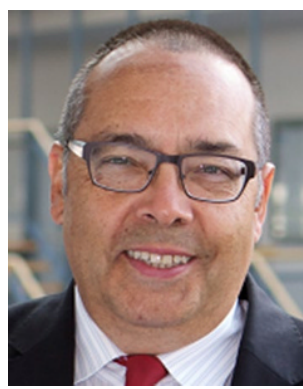

Prof. Dr. Helmut Krcmar

ist Leiter der Forschungsgruppe Krcmar an der Technischen Universität München. Zusätzlich ist er Beauftragter des Präsidenten für die Gesamtentwicklung des TUM Campus Heilbronn.

${ }^{1}$ Technische Universität München, Fakultät für Informatik, Forschungsgruppe Krcmar, München, Deutschland später durchlebte das Internet eine Renaissance, die heutzutage allgemein als Übergang zum Web 2.0 gesehen wird [5]. Sie ist charakterisiert durch die Ablösung statischer Internetseiten, die nur gelegentlich überarbeitet und angepasst wurden, durch interaktive Webanwendungen, die Endnutzern erstmals den Konsum und die Bereitstellung eigener Inhalte erlaubte.

Die Technologien des Web 2.0 bildeten die Grundvoraussetzungen für die ersten Web-Foren, in denen Nutzer eigene Beiträge schreiben und ohne die Webseite neu zu laden, die Kommentare anderer Nutzer einsehen konnten. Diese für die damalige Zeit unkonventionelle Möglichkeit digitaler Interaktion inspirierte Gründer, das Web 2.0 zur Lösung bestehender Probleme zu nutzen. Die Gründer des heute weithin bekannten Mobilitätsdienstleisters BlaBlaCar erkannten das Potenzial des Web 2.0, um dezentral organisierte Mitfahrgelegenheiten auf einer Webseite zu bündeln. Auf Basis dieser Idee entwickelten sie eine Plattform, auf der Nutzer individuelle Präferenzen zu ihrer Fahrt angeben und sofort bestmögliche Angebote einsehen konnten. Nach einer Buchung konnten sie sich zudem mit Fahrern in einem WebChat austauschen, um z. B. das örtliche Treffen zu koordinieren und schließlich nach abgeschlossener Fahrt eine Bewertung abzugeben. BlaBlaCar war somit in der Lage, den gesamten Prozess zwischen Nachfrage und Angebot durch den Einsatz des Web 2.0 abzubilden [6].

Im Jahr 2009 entwickelten die Gründer von Uber aus der Vielzahl von Web-Foren die Idee, lokale Fahrdienste über einen digitalen Marktplatz anzubieten. Wie zuvor BlaBlaCar, versuchte auch Uber die neuen Potenziale des Web 2.0 anhand eines Plattformmodells abzubilden. Der dynamische Austausch von Daten und Webseiteninhalten ermöglichte es Uber, die eingehenden Anfragen von Passagieren effizient den zur Verfügung stehenden Fahrern zuzuordnen. Durch diese schnelle und effektive Zuordnung konnten sich die ersten digitalen Plattformen im Mobilitätsbereich von Wettbewerbern wie örtlichen Taxiunternehmen abgrenzen [7].

Auch etablierte Unternehmen in der Mobilitätsbranche - wie z. B. Automobilhersteller - realisierten die Vorteile des Web 2.0. Anwendungsgebiete der Technologien wurden dabei hauptsächlich im Rahmen der bestehenden Produktionslogik, also Automobile kosteneffizient und qualitativ hochwertig zu bauen, gesucht. So nutzte ein Automobilhersteller die neuen Funktionalitäten des Web 2.0, um das Kundenfeedback aufzunehmen, zu beantworten und in die Verbesserung eigener Prozesse einfließen zu lassen. Anstatt ein neues Geschäftsmodell auf der Grundlage neuer Technologien zu entwickeln, wurde das bestehende Geschäftsmodell zielgerichtet verbessert.

\section{Phase 2: Smartphones}

Die Vorstellung des iPhone im Jahr 2007 markiert einen Meilenstein technologischer Innovationen. Die grundlegende Innovation des iPhones sind sogenannte Applikationen. Diese werden von unabhängigen Entwicklern angeboten und erweitern die Funktionsweise des Smartphones durch beispielsweise Nachrichtendienste, Kalenderlösungen oder Spiele [8]. Ein weiterer Vorteil sind die eingebauten Sensoren, welche es Nutzern ermöglichen, 
ihren Standort zu ermitteln, das Internet mobil zu nutzen oder kontaktlose Zahlungen vorzunehmen. Kombiniert man die Potenziale der eingebauten Sensoren mit der Möglichkeit, eigene Applikationen zu entwickeln, ergeben sich innovative Möglichkeiten.

Uber bildet ein Paradebeispiel dafür, wie die Kombination neuartiger Technologien des Web 2.0 und des Smartphones ein komplett neues, plattformbasiertes Geschäftsmodell ermöglichen. Ungleich BlaBlaCar, die im Fernverkehr darauf vertrauen können, dass Kunden frühzeitig eine Mitfahrgelegenheit planen und buchen, womit ein stationärer Zugriff über die Plattform/BlaBlaCar ausreicht, ist die Nachfrage im örtlichen Verkehr deutlich volatiler. So besteht ein Großteil der über Uber gebuchten Mobilitätsdienstleistungen aus spontanen Fahrten zu Geschäftsmeetings oder sporadischen Treffen. Die Abdeckung dieser Mobilität und Volatilität war nur möglich, indem Uber die dynamischen Inhalte des Web 2.0 in eine Smartphone basierte Applikation migrierte und die GPS-Sensoren und das mobile Internet nutzte, um die Standorte von Fahrern und Kunden auszuwerten. Hierdurch konnte Uber mit Taxiunternehmen konkurrieren und den Anruf in der Taxizentrale durch einen Knopfdruck per Applikation ersetzen. Die schnelle Ausbreitung der Smartphones durch deren zunehmende Bezahlbarkeit und einfache Bedienbarkeit sorgte schließlich dafür, dass die benötigte Infrastruktur weit genug verbreitet war, um Ubers Services skalieren zu können [7].

Die bessere Planbarkeit der Mitfahrgelegenheiten ermöglichte es BlaBlaCar zwar schon vor 2007 eine Plattform aufzubauen, jedoch wurden auch hier die Vorteile des Smartphones genutzt, um das Geschäftsmodell weiterzuentwickeln. Wie bei Uber gelang es BlaBlaCar, über eine SmartphoneApplikation auch kürzere Fahrten zu vermitteln. Wichtiger für das Unternehmen war jedoch, über das im Smartphone integrierte GPS, die Treffen zwischen (Mit-)Fahrern besser zu koordinieren und zu kontrollieren, ob Fahrten tatsächlich unternommen wurden. Damit konnte der Vermittlungsprozess bis zur Abwicklung erweitert werden, wodurch die Plattform an Qualität, Seriosität und Kundenbindung gewann [2,9].

Etablierte Automobilbauer erkannten die Vorteile mobiler Endgeräte ebenso und integrierten die Sensoren, das benutzerfreundliche Design und die Internetkonnektivität als Subsysteme in ihre Automobile. So ist der Bordcomputer mit dem GPS-Navigationsgerät, der Mediaverwaltung basierend auf mobilem Internet und der Freisprechanlage durch SmartphoneKopplung heutzutage Standard. Anders als bei Uber oder BlaBlaCar wurden die Innovationen aber nicht mit denen des Web 2.0 kombiniert oder als Erweiterung genutzt. Stattdessen wurden sie präzise in einem bestimmten Bereich der bestehenden Produktion angewandt, um das eigene Produkt gezielt zu verbessern.

\section{Phase 3: Cloud-Computing}

Die weltweite Integration mobiler Endnutzer hatte einen massiven Bedarf an Prozessorkapazitäten zur Folge und ebnete damit den Weg für das Cloud-
Kernthese 1

Plattformunternehmen

explorieren Technologiepotenziale ergebnisoffen, während etablierte Unternehmen neue Technologien gezielt und in begrenztem Umfang einsetzen, um konkrete Probleme zu lösen. 


\section{Kernthese 2}

\section{Plattformunternehmen nutzen}

neue Technologien, um

Kunden und Geschäftspartner

aktiv in die Wertschöpfung

einzubeziehen, während

etablierte Unternehmen Wert-

schöpfungsprozesse innerhalb

des eigenen Unternehmens

optimieren.
Computing. Cloud-Computing beschreibt über das Internet zugänglich gemachten Speicherplatz, Rechenleistung oder Anwendungssoftware. Zentraler Vorteil der verschiedenen Cloud-Service-Modelle ist, dass Unternehmen weniger eigene Infrastruktur vorhalten müssen, sondern bestehende Server bedarfsbasiert nutzen und abrechnen können.

Die Skalierung nativer Plattformen durch die zuvor beschriebenen Innovationen führte zu einem Kostenanstieg für die Bereitstellung und Instandhaltung der Services. Uber und BlaBlaCar hatten ihre zentralen Systeme zur Bedienung der Datenanfragen monolithisch angelegt, d. h. alle Services von einem einzigen System in einem unternehmenseigenen Rechenzentrum verarbeitet. Die Masse und Volatilität der Anfragen in Kombination mit den Kosten der Instandhaltung und langfristigen Skalierung bewogen Uber und BlaBlaCar, externe Cloud-Services zu integrieren und proprietäre Systeme in die Cloud zu migrieren. In Kombination mit bestehenden Technologien konnten Services schneller und zuverlässiger in der gesamten Welt angeboten werden, da auf ein breites Netz bestehender Datencenter zurückgegriffen werden konnte $[10,11]$.

Etablierte Automobilhersteller nutzten die Cloud in ähnlicher Weise, um die Kosten bestehender IT-Systeme zu verringern oder die Flexibilität von IT-Dienstleistungen zu erhöhen. Gerade in Kombination mit OutsourcingProgrammen bot die Integration externer Cloud-Services vielfältige Möglichkeiten, die eigene IT zu verschlanken und den Fokus auf das eigentliche Produkt zu legen. Häufige Einsatzgebiete für Cloud-Dienste liegen dabei im Bereich administrativer Programme wie der Buchhaltung oder Personalverwaltung.

\section{Phase 4: Data Analytics}

Die Integration von Nutzern in die Datengenerierung durch das Web 2.0 in Kombination mit der zentralen Datenhaltung und -verarbeitung über die Cloud eröffnet neue Möglichkeiten in der Datenverwertung und -analyse. Data Analytics umschreibt die Analyse und Auswertung großer Datenmengen zur Generierung von Erkenntnissen und zur Entscheidungsunterstützung. Im Rahmen statistischer Auswertungen wird dabei vor allem versucht, Muster in großen Datenmengen zu finden, die auf ein bestimmtes Verhalten von verschiedenen Stakeholdern und Entitäten in der Unternehmensumgebung hinweisen. Entsprechende Erkenntnisse können dem Management bei der Entscheidungsfindung helfen, aber auch unmittelbar über eine effiziente Gestaltung von Prozessen und Projekten die Dienstleistungsqualität erhöhen.

Die Bereitstellung eines qualitativ hochwertigen und auf individuelle Nutzerbedürfnisse abgestimmten Services wird mit der Anzahl an Nutzern zunehmend komplexer und erschwert die Entscheidungsfindung des Managements. Um dennoch qualitativ hochwertige Services bereitzustellen, haben die Betreiber nativer Plattformen erkannt, dass sich jede einzelne Technologie (Web 2.0, Smartphones, Cloud) mit Data Analytics kombinieren lässt. Die Auswertung von Nutzungsdaten erlaubt es Uber wie auch BlaBlaCar, 
das Nutzungsverhalten von Fahrern und Passagieren besser analysieren zu können. Hierdurch können zum einen Services proaktiv optimiert werden und zum anderen kann die Plattform basierend auf den Daten strategisch weiterentwickelt werden. Uber zum Beispiel versorgt Nutzer gezielt mit Angeboten und Hinweisen, sobald sie bemerken, dass das Engagement auf der Plattform nachlässt oder von gewohnten Nutzungszeiträumen abweicht.

Auch etablierte Automobilbauer integrierten Data Analytics früh, um aus den eigenen Daten Rückschlüsse auf mögliche Produktoptimierungen zu ziehen. Ungleich nativer Plattformen, welche hauptsächlich nutzergenerierte Daten speichern, besitzen etablierte Unternehmen eher produkt- oder prozessbasierte Datensätze. Ein Automobilbauer überwacht z. B. kontinuierlich, wie schnell ein Band läuft, in welchen Zyklen neue Teile bestellt werden müssen oder wie effizient einzelne Mitarbeiter bestimmte Fertigungsschritte ausführen. Entsprechend unterstützen Auswertungen die Verbesserung bestehender Prozesse und Produkte, aber weniger die Entwicklung von nutzerorientierten Services und plattformbasierten Geschäftsmodellen.

\section{Unterschiede im Umgang mit neuen Technologien}

\section{Exploration von Technologiepotenzialen vs. gezielte Optimierung}

Der Umgang nativer Plattformen mit neuen Technologien ist geprägt durch einen ergebnisoffenen Ansatz der Exploration von Technologiepotenzialen [12]. Hierdurch können Unternehmen mit limitierten Ressourcen einen Markt zwischen Angebot und Nachfrage durch eine digitale Plattform abbilden. Dem Plattformbetreiber kommt dabei zugute, dass die Kombination verschiedener Technologien ein neues Plattformökosystem schafft, in das mehrere Parteien integriert werden können. Uber zum Beispiel wurde durch seine begrenzten Ressourcen dazu gezwungen, eine rein softwarebasierte Plattform aufzubauen. Die Kombination aus Web 2.0, mobilen Endgeräten sowie einer cloudbasierten Datenverwaltung erlaubte es Uber, die bestehende Nachfrage nach Mobilitätsservices, die zuvor durch Taxen und den öffentlichen Nahverkehr abgedeckt wurde, über einen digitalen Service zu bündeln.

Anders als Betreiber nativer Plattformen können etablierte Unternehmen Werkzeuge und Ressourcen einem geplanten Ziel entsprechend wählen, ohne Potenziale neuer Technologien explorieren zu müssen. Dementsprechend haben viele dieser Unternehmen die neuen Technologien wie das Web 2.0 oder mobile Endgeräte gezielt eingesetzt, um bestehende Probleme zu lösen. Da die eigene Nachfrage aber bereits durch ein bestehendes Produkt abgedeckt wird, bestand keine Notwendigkeit, innovative Technologien zu kombinieren, um neue Wege zu gehen. Wie an dem Beispiel des Automobilbauers zuvor zu erkennen war, wurde jede einzelne Technologie zielgerichtet in Teilbereichen des Unternehmens eingesetzt.

\section{Kernthese 3}

Durch die Kombination verschiedener Technologien schaffen Plattformunternehmen neue technologische und ökonomische Einflussfaktoren, die etablierte Unternehmen unter Druck setzen. 


\section{Zusammenfassung}

Wenn man sich die Nutzung von neuen Technologien zwischen Plattformen und etablierten Unternehmen ansieht, fallen drei Besonderheiten auf:

1. Digitale Plattformen explorieren das Nutzungspotenzial deutlich mehr als etablierte Unternehmen.

2. Digitale Plattformen nutzen neue Technologien, um Kunden und Partner in den Wertschöpfungsprozess mit einzubeziehen.

3. Digitale Plattformen verändern durch die Kombination neuer Technologien technologische und ökonomische Bedingungen für etablierte Unternehmen.

\section{Plattformlogik vs. Produktionslogik}

Die Innovationskraft nativer Plattformen liegt nicht nur in der Kombination neuer Technologien, sondern auch in der Struktur des Geschäftsmodells. Native Plattformen bewältigen die Wertschöpfung nicht alleine, sondern sehen ihre Aufgabe in der Orchestrierung einer effizienten Verknüpfung von Angebot und Nachfrage [13]. Wie bereits angedeutet, ist das Ergebnis der Nutzung von Uber und einem Taxi identisch - die Fahrt von einem Ort A zu einem Ort B. Die Nutzung der Uber-Applikation verspricht Nutzern allerdings einen schnelleren, einfacheren und vor allem kostengünstigeren Zugang zu einem Fahrer in der Nähe, der keine zusätzlichen Informationen benötigt.

Etablierte Unternehmen sind dagegen in ihrer Handlungsweise an etablierte Strukturen gebunden. Der als Beispiel herangezogene Automobilbauer gründet seinen Erfolg auf der eigenen Produktionslogik, die die Konstruktion und den Verkauf qualitativ hochwertiger Autos an seine Kunden als Ziel definiert. Entsprechend setzen Automobilbauer neue Technologien gezielt und separat ein, um bestimmte Eigenschaften und Facetten der Produktion eines Automobils zu verbessern. Das Web 2.0 zum Beispiel, um Kundenfeedback schneller aufzunehmen, oder Sensoren mobiler Endgeräte, um den Bordcomputer zu erweitern.

\section{Diffusion vs. Adaption}

Die Etablierung des Paradigmas der Orchestrierung hatte direkte Auswirkungen auf die Diffusion technologischer und ökonomischer Einflussfaktoren [14]. So machten Plattformen wie Uber oder BlaBlaCar den technologischen Ansatz der Microservices zum De-facto-Standard für Plattformen, indem sie ihre monolithischen Systeme in viele modular aufgebaute Subsysteme aufbrachen. Ökonomische Einflüsse drücken sich zum Beispiel in der heute weitverbreiteten „Gig Economy“ aus, die durch Uber berühmt wurde und in der Privatpersonen zu bezahlten Dienstleistern wurden.

\section{Abb. 1 Umgang nativer Plattformen und etablierter Unternehmen mit neuen Technologien}

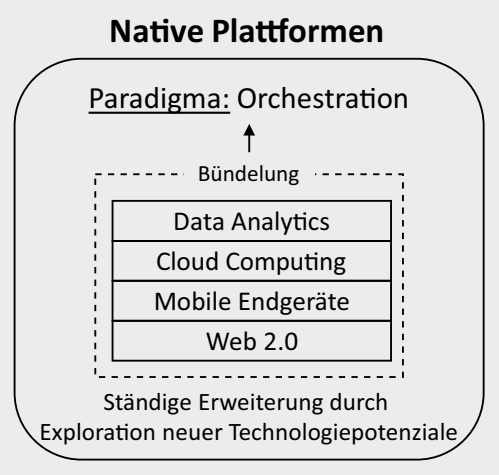

Etablierte Unternehmen

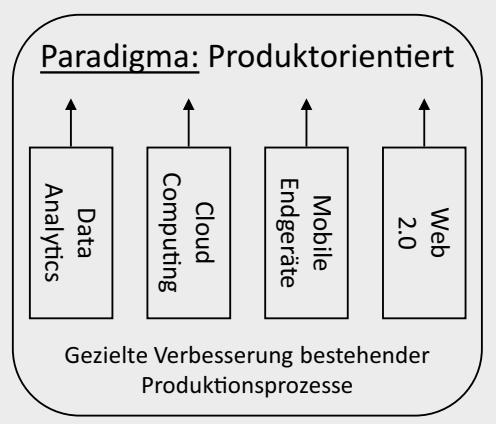


Die akute Veränderung durch das neue Paradigma zwingt etablierte Unternehmen zu einem Umdenken hinsichtlich der Entwicklung eigener Plattformen. So gefährdet die beschriebene Gig Economy die Absatzplanungen traditioneller Automobilhersteller, indem der Pro-Kopf-Bedarf an Fahrzeugen tendenziell sinkt. Diese und weitere Entwicklungen (z. B. Elektrifizierung) initiieren einen Willen zur Adaption und erhöhen die Risikobereitschaft für eine eigene Transformation hin zu einem Plattformmodell.

\section{Fazit}

Die Entstehung nativer digitaler Plattformen wird durch einen ergebnisoffenen Umgang mit neuen Technologien wie dem Web 2.0, Smartphones, Cloud und Data Analytics beeinflusst. Die Herangehensweise unterscheidet sich fundamental von etablierten Unternehmen, die neue Technologien im Rahmen fester Strukturen gezielt und punktuell einsetzen (s. Abb. 1).

Während native Plattformen durch eine gezielte Exploration von Technologiepotenzialen verschiedene Innovationen zu einer integrierten Plattform kombinieren und damit einen bestehenden Markt orchestrieren, adressieren etablierte Unternehmen nur einzelne Optimierungen im Rahmen der bestehenden Produktionslogik. Um nicht den Anschluss an die Plattformökonomie zu verlieren, sollten etablierte Unternehmen deshalb nicht nur Technologien und ihre Kombinationen stärker explorieren, sondern auch das eigene Geschäftsmodell kontinuierlich hinterfragen und entwickeln. Zudem müssen etablierte Unternehmen zunehmend Kunden und Geschäftspartner aktiv in den Wertschöpfungsprozess einbeziehen, um mit plattformbasierten Geschäftsmodellen konkurrieren zu können.

Funding. Open Access funding enabled and organized by Projekt DEAL.

Open Access Dieser Artikel wird unter der Creative Commons Namensnennung 4.0 International Lizenz veröffentlicht, welche die Nutzung, Vervielfältigung, Bearbeitung, Verbreitung und Wiedergabe in jeglichem Medium und Format erlaubt, sofern Sie den/die ursprünglichen Autor(en) und die Quelle ordnungsgemäß nennen, einen Link zur Creative Commons Lizenz beifügen und angeben, ob Änderungen vorgenommen wurden.

Die in diesem Artikel enthaltenen Bilder und sonstiges Drittmaterial unterliegen ebenfalls der genannten Creative Commons Lizenz, sofern sich aus der Abbildungslegende nichts anderes ergibt. Sofern das betreffende Material nicht unter der genannten Creative Commons Lizenz steht und die betreffende Handlung nicht nach gesetzlichen Vorschriften erlaubt ist, ist für die oben aufgeführten Weiterverwendungen des Materials die Einwilligung des jeweiligen Rechteinhabers einzuholen.

Weitere Details zur Lizenz entnehmen Sie bitte der Lizenzinformation auf http://creativecommons.org/licenses/by/4.0/deed.de.

\section{Handlungsempfehlung}

- Technologiepotenziale explorieren: Explorieren technologischer Potenziale und Integration dieser in die eigene Wertschöpfung

- Geschäftsmodelle innovieren: Berücksichtigung einer ständigen Evolution des Geschäftsmodells, entsprechend des Marktes, des Wettbewerbs und aufkommender technologischer Innovationen

- Paradigma der Orchestration adaptieren: Einbindung und Orchestrierung verschiedener Interessengruppen über eine digitale Plattform, um eine gemeinsame Wertschöpfung zu ermöglichen 


\section{Literatur}

[1] Evans, P. C., \& Gawer, A. (2016). The rise of the platform enterprise: a global survey. The emerging platform economy series. New York: The Emerging Platform Economy Series.

[2] Hein, A., et al. (2019). The emergence of native multi-sided platforms and their influence on incumbents. Electronic Markets, 29(4), 631-647.

[3] Hein, A., et al. (2020). Digital platform ecosystems. Electronic Markets, 30(1), 87-98.

[4] McIntyre, D. P., \& Srinivasan, A. (2017). Networks, platforms, and strategy: emerging views and next steps. Strategic Management Journal, 38(1), 141-160.

[5] O'Reilly, T. (2007). What is Web 2.0: Design patterns and business models for the next generation of software. Communications \& strategies, 65 (1), 17.

[6] BlaBlaCar (2018). Fail. Lern. Succeed. https://www.blablacar.com/blog/insidestory/fail-learn-succeed. Zugegriffen: 1. Febr. 2018.

[7] Hein, A., Böhm, M., \& Krcmar, H. (2018). Tight and loose coupling in evolving platform ecosystems: the cases of Airbnb and Uber. In Proceedings of the 21st International Conference on Business Information Systems. Berlin: Springer.

[8] Eaton, B., et al. (2015). Distributed tuning of boundary resources: the case of Apple's iOS service system. MIS Quarterly, 39(1), 217-243.

[9] Rose, I., \& Wheeler, M. (2017). How BlaBlaCar created a global transport network. https://www.bbc.com/news/business-38597504. Zugegriffen: 1. Febr. 2018.

[10] Reinhold, E. (2016). Rewriting Uber engineering: the opportunities microservices provide. https://eng.uber.com/building-tincup/. Zugegriffen: 1. Febr. 2018.

[11] Haddad, E. (2015). Service-oriented architecture: scaling the Uber engineering codebase as we grow. https://eng.uber.com/soa/. Zugegriffen: 1. Nov. 2017.

[12] Baker, T., \& Nelson, R. E. (2005). Creating something from nothing: resource construction through entrepreneurial bricolage. Administrative Science Quarterly, 50(3), 329-366.

[13] Parker, G., et al. (2016). Platform revolution: How networked markets are transforming the economy and how to make them work for you. New York: W. W. Norton.

[14] Rogers, E. M. (2003). Diffusion of innovations. Bd. 5. New York: The Free Press. 
Hier steht eine Anzeige.

\section{Springer}

